\title{
Hybrid procedure for orbital venous malformation in the endovascular operation room
}

ACO Cheng ${ }^{1,2}$, EYM Li ${ }^{1,2}$, TCY Chan ${ }^{1,2}$ ACW Wong ${ }^{1,2}$, PCM Chan ${ }^{3}$, WWL Poon ${ }^{4}$, DHS Fung ${ }^{4}$ and HKL Yuen ${ }^{1,2}$

\begin{abstract}
Purpose To describe a hybrid procedure for orbital venous malformation in the endovascular operating room (EVOR). Methods Five consecutive patients with venous malformation in the periocular and orbital region were included. All patients received a one-stage direct puncture venogram, image-guided glue injection, and surgical resection in the EVOR equipped with a biplane digital subtraction angiography system (BDSAS).

Results The mean age at the time of operation was 37.4 years (range, 22-69 years). The mean operative time was $193 \mathrm{~min}$ (range, 138-324 min). No intraoperative complications were noted. The mean follow-up duration was 18.8 months (range, 10-24 months). Three patients had complete removal of the vascular lesions. At the latest follow-up, no recurrence of symptoms related to the lesions was noted. All patients had an uneventful recovery and satisfactory outcome.

Conclusions The hybrid procedure of orbital venous malformation in the EVOR is a novel application in ophthalmology. It is a safe and well-controlled procedure with real-time high-quality BDSAS surveillance to facilitate surgical resection. Its success requires collaboration between the interventional radiologist, the surgeon, and the ophthalmologist.
\end{abstract}

Eye (2015) 29, 1069-1075; doi:10.1038/eye.2015.72; published online 29 May 2015

\section{Introduction}

Orbital venous malformations are abnormal developments of the orbital venous system that have variable communication with the systemic venous circulation. Distensible lesions have a direct and rich communication with the venous system, whereas non-distensible anomalies have a minimal systemic connection. ${ }^{1}$ Apart from spontaneous hemorrhage or thrombosis, orbital venous malformations can lead to severe pain, proptosis, diplopia, visual disturbance, and cosmetic disfigurement. A variety of techniques including carbon dioxide laser ablation, percutaneous sclerotherapy, surgical resection, and embolization followed by excision have been attempted. ${ }^{2,3}$ As there has been no standardized treatment for these lesions and treatment outcomes are sometimes not as satisfactory, most cases are observed conservatively. Surgical interventions are possible in some centers for selected cases, especially when complications arise. Preoperative or intraoperative venography and fluoroscopy have been used to assist the surgical excision of orbital venous malformations. ${ }^{2,4,5}$ However, limitations in interventional radiology support and intraoperative visualization technology are often major obstacles for a safe and complete surgical removal.

The endovascular operating room (EVOR) is a hybrid operating room-angiography suite (Figure 1). It is a place that meets the stringent requirements of an operating theater while harboring the facilities of an interventional radiology room. ${ }^{6}$ With the ability to perform real-time imaging and three-dimensional reconstruction, these sophisticated high-end imaging capabilities greatly facilitate complex endovascular operations. The hybrid operating room has been used successfully for diagnostic and therapeutic vascular procedures in cardiovascular and cerebrovascular surgeries. 7,8 This case series reported a combined endovascular and surgical management of orbital venous malformations in the EVOR, which is a novel application of this technology.
${ }^{1}$ Hong Kong Eye Hospital, Hong Kong, China

${ }^{2}$ Department of Ophthalmology and Visual Sciences, The Chinese University of Hong Kong Hong Kong, China

${ }^{3}$ Department of Surgery, Queen Elizabeth Hospital, Hong Kong, China

${ }^{4}$ Department of Radiology and Imaging, Queen Elizabeth Hospital, Hong Kong, China

Correspondence: HKL Yuen, Hong Kong Eye Hospital, 147K Argyle Street, Kowloon, Hong Kong, China Tel: +852 27623000 ; Fax: +852 27626305 E-mail: hunterklyuen@ gmail.com

Received: 25 September 2014

Accepted in revised form: 27 March 2015

Published online: 29 May 2015

Presentation: Free paper presentation at the American Academy of Ophthalmology Annual Meeting, New Orleans, Louisiana, November 2013 


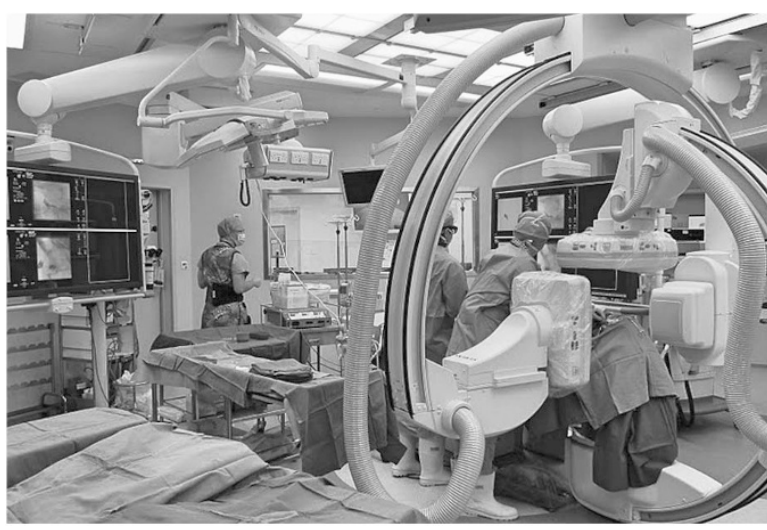

Figure 1 The setting of an endovascular operation room.

\section{Materials and methods}

A retrospective, non-comparative clinical study to evaluate the management of orbital venous malformation in the EVOR was conducted from September 2011 to December 2013. This study conformed to the tenets of the Declaration of Helsinki and was approved by the Institutional Review Board at Kowloon Central Cluster, Hospital Authority, Hong Kong.

Consecutive patients who underwent surgery in the Jockey Club Endovascular \& Minimal Access Operating Centre in Queen Elizabeth Hospital, Hong Kong, were identified from the operating theater master record. Medical records of the relevant patients were retrieved and reviewed. Inclusion criteria included patients with venous malformation in the periocular and orbital region, patients who had undergone an intraoperative venogram and injection of tissue glue into the vascular lesion by interventional radiologists, and patients who had undergone open surgical removal of the lesion by ophthalmologists and vascular surgeons. All cases must have been managed in the specified established EVOR in a single operative session. Patients who had undergone sclerosant or glue injection and open surgery in separate settings were excluded from this study.

All patients in this series were initially assessed by ophthalmologists in the Orbital and Oculoplastic Clinic at the Hong Kong Eye Hospital. Individuals with symptomatic orbital vascular lesions at the periocular area who were keen on undergoing surgical resection were referred to the Vascular Clinic at Queen Elizabeth Hospital and assessed by vascular surgeons and interventional radiologists. All patients underwent contrast-enhanced magnetic resonance imaging (MRI) or computed tomography (CT) and Doppler ultrasound study when necessary to confirm the diagnosis of venous malformation and assess its extent. A joint intervention was planned and arranged at the EVOR if the patient decided to undergo surgery. All patients gave their informed consent in the present study. Informed consent for publication of the clinical photos before and after operation was obtained from each patient in the current study.

The EVOR is a special setup in which a biplanar digital subtraction angiographic system (BDSAS) (Artis zee biplane system, Siemens, Erlangen, Germany) is installed in a standard operating theater with positive pressure airflow, standard sterilization protocols, infection control standards, and a full range of surgical instruments available. In this series, all cases underwent an intraoperative direct puncture venogram using BDSAS to confirm the extent of the lesion and the draining pattern of the vascular lesions, especially any intracranial vascular communication, by interventional radiologists. For those orbital venous malformations involving multiple sites, small-sized lesions for which surgical excision was deemed unnecessary, sclerotherapy with 5\% ethanolamine was performed. For larger-sized lesions, a mixture of histoacryl glue (n-butyl cyanoacrylate) (B. Braun Surgical SA, Rubi, Spain) and lipiodol (Guerbet, Roissy CDG, France), which is a glue-radiopaque dye mixture, was injected to solidify the lesion, followed by surgical resection (transconjunctival or transcutaneous) by ophthalmologists subspecialized in orbital and oculoplastic surgery and by vascular surgeons. For superficial lesions, a venogram via percutaneous puncture was performed, whereas for deep-sitting vascular lesions surgical dissection was performed to expose the lesion prior to the venogram to enhance the accuracy and safety of the procedure. Multiple puncture sites with a glue-dye mixture injection were required for multi-loculated lesions to ensure more complete glue embolization of the lesion. A supplementary venogram, as well as injection of the glue-dye mixture, was performed when necessary, along with surgical dissection. All procedures were performed in the EVOR without transfer of the patient. Patients were followed up at postoperative day 1 , week 1 , week 2 , and month 1 , and 3 monthly thereafter.

The main outcome measures were complete removal of the vascular lesion and correction of disfigurement. Intraoperative complications, especially intracranial spillage of sclerosant or tissue glue, cerebrovascular events, and excessive bleeding, were assessed. Postoperatively, adverse events including visual loss, ocular dysmotility, wound infection, secondary hemorrhage, tissue necrosis, and unsightly scar were evaluated.

\section{Results}

A total of five patients, including three females and two males, underwent treatment of orbital venous 

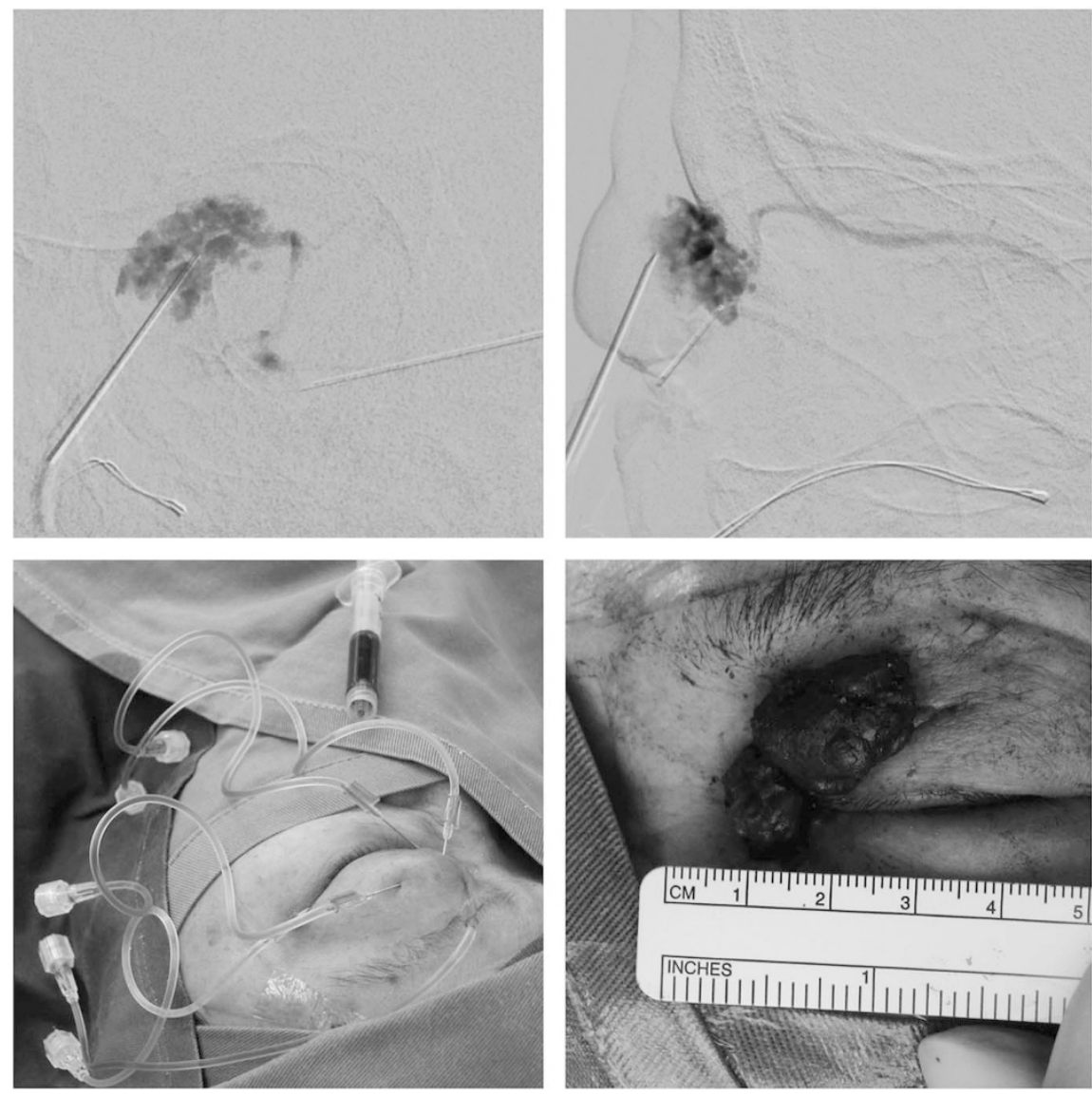

Figure 2 Clinical photos of Case 1. (Top left) Injection of tissue glue and contrast mixture was visualized and localized with the anterior-posterior view under the biplanar digital subtraction angiographic system. (Top right) The corresponding lateral view under the biplanar angiographic system. (Bottom left) Injection of tissue glue and contrast mixture at various sites because of the multiloculated nature of the venous malformation. (Bottom right) The glued venous malformation was excised.

malformation in the EVOR. All patients were operated upon under general anesthesia. The mean age was 37.4 years (range 22-69 years). The mean duration of follow-up was 18.8 months (range 10-24 months). The mean operative time was $193 \mathrm{~min}$ (range 138-324 min). All patients except two had complete removal of the vascular lesion. All patients were satisfied with the cosmetic improvement. At the latest follow-up, no recurrence of symptoms related to the lesions was noted. No intraoperative complications were documented. Postoperatively, none suffered from visual loss from the surgery. One patient with a massive lesion had mild limitation in abduction on postoperative assessment. No other adverse events such as wound infection, secondary hemorrhage, tissue necrosis, and unsightly scar were noted.

\section{Case reports}

Case 1 A 69-year-old woman was referred to our hospital for left orbital mass involving the upper and lower eyelids. Over the last few decades, she had received multiple interventions including sclerotherapy and surgical resection. However, the lesion remained and increased in size over the years, and she suffered from left mechanical ptosis, severe periorbital pain, and discomfort, resulting in psychological disturbance. Contract CT and MRI of the orbit showed a low flow infiltrative vascular lesion of both eyelids suggestive of a venous malformation. The operation was carried out in the EVOR. The lower lid venous malformation was managed by injecting sclerosant into the lesion guided by BDSAS. The upper lid lesion was excised through a transverse skin incision after glue embolization guided by the BDSAS. Multiple injection sites were needed because of the multiple loculations of the venous malformation. Histopathology confirmed a multi-loculated venous malformation. At 18 months' follow-up, the patient was seen to be experiencing no discomfort and no recurrence of ptosis. She was satisfied with the surgical outcome (Figure 2).

Case 2 A 23-year-old woman described a progressive swelling in her left upper lid over a 2-year period. This 

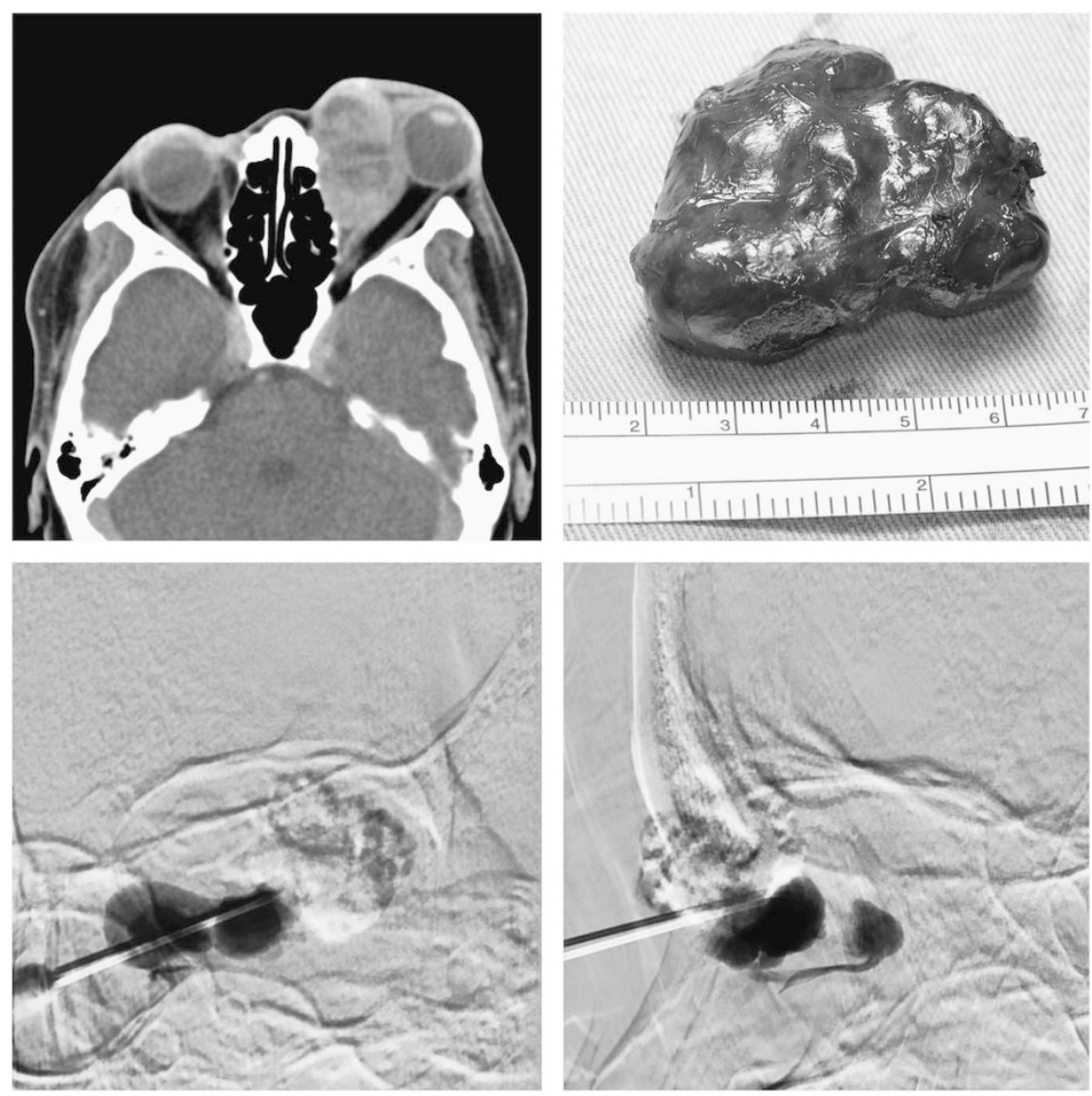

Figure 3 Clinical photos of Case 3. (Top left) Computed tomography showing a large contrast-enhancing lesion with posterior orbital extension. (Top right) The glued venous malformation was excised after embolization. (Bottom left) Injection of tissue glue and contrast mixture was visualized and localized with the anterior-posterior view under the biplanar digital subtraction angiographic system. (Bottom right) The corresponding lateral view under the biplanar angiographic system.

was associated with marked cosmetic blemish and mild discomfort. Her vision and ocular motility remained normal. A CT scan showed a vascular malformation extending from the left upper lid posteriorly to the trochlear region. Her symptoms gradually increased, and she underwent surgical excision of the venous lesions at the EVOR. Intraoperative direct puncture venogram and glue injection were performed by radiologists. This was followed by sub-brow skin incision over the lesion. With the aid of BDSAS-guided glue injection, the vascular lesion was subsequently excised as much as possible up to the trochlea region, preserving the superior oblique muscle tendon. Histology showed ectatic thin-walled vascular channels consistent with venous malformation. She was free of pain, and there was no evidence of recurrence at the recent follow-up after 10 months.

Case 3 A 38-year-old woman had a left upper lid vascular lesion since the age of 24 years. She had received incomplete surgical excision of the lesion 10 years ago in China, which was histologically revealed to be dysplastic venous channels. Subsequently she was noted to have progressive proptosis, ocular dysmotility, and compressive optic neuropathy. MRI of her orbit showed a contrast-enhancing extraconal mass at the antero-medial aspect of her left globe. It displaced the left medial rectus muscle and the globe laterally. She received sclerosant injection to her left orbital venous malformation, but the clinical response was transient. In view of the progressive deterioration in visual function, repeated excision was carried out in the EVOR. BDSAS-guided glue injection was performed before panorbitotomy, combining lateral canthotomy and inferior cantholysis, removal and subsequent replacement of the lateral orbital rim, transconjunctival and trancaruncular incision, and vertical eyelid splitting. The solidified part of the vascular lesion was excised with a clear delineated dissection plane; yet the deeper part of the lesion did not take up sufficient glue with the superficial puncture and glue injection. In view of the extensive lesion size, a repeated venogram with additional glue injection under BDSAS guidance was supplemented (Figure 3). Complete 
excision was achieved, and the follow-up orbital CT performed 18 months after excision did not reveal any recurrence. Despite the poor left visual acuity as the consequence of prolonged optic nerve compression and established optic atrophy, her vision improved from hand movement to 6/60 after operation. There was mild limitation in left eye abduction but the patient has no diplopia, and there was no clinical recurrence with satisfactory cosmetic outcome.

Case 4 A 22-year-old man had a left conjunctival vascular lesion since 10 years of age. He noted that the mass was gradually increasing in size and causing significant cosmetic disfigurement. On MRI of the orbit, a multi-loculated intraconal lesion was noted at the inferomedial aspect of the left orbit. The lesion extended anteriorly to the preseptal space involving the inferior eyelid and posteriorly to the intraconal orbital space with features suggestive of a slow-flow orbital venous malformation. The lesion was managed in the EVOR collaboratively by radiologists, vascular surgeons, and ophthalmologists. Contrast injection followed by glue injection under BDSAS guidance was performed through insertion of the catheter to the inferior lid lesion. The glued venous malformation together with the feeder vessels was excised through a transconjunctival approach. The lesion was noted to have infiltrated the inferior rectus muscle, and the latter was preserved. A small portion of the most posterior intraconal component was left in view of surgical risk outweighing the benefits. Histopathology showed dysplastic venous channels with lymphatic components. There was no clinical recurrence noted 2 years after the operation despite a small residual intraconal venous malformation on follow-up MRI. His visual acuity remained good with no ocular dysmotility and disfigurement.

Case 5 A 35-year-old man presented with an intermittent left upper lid mass since 6 months. The mass increased in size after valsalva maneuver and caused mechanical ptosis and periocular discomfort, but there was no proptosis or ocular dysmotility. He had a ruptured left eyeball repaired over 10 years ago, with subsequent poor visual acuity. Because of the cosmetic disfigurement and discomfort, surgical resection at the EVOR was performed. Dynamic contrast CT of the orbit showed a lobulated nodular lesion in the pre-septal compartment of the left orbit enlarging on prone valsalva maneuver. The operation was carried out in the EVOR. Superficial dissection was performed through a lid crease incision until the vascular malformation was exposed. This was followed by a direct puncture venogram, followed by contrast and glue injection into the lesion under BDSAS surveillance to avoid spillage from the superior ophthalmic vein to the cavernous sinus (Figure 4). After embolization, the lesion was removed completely en bloc. Histopathology of the specimen showed dilated vascular channels compatible with venous malformation. The patient remained asymptomatic with no recurrence at follow-up 2 years later.

\section{Discussion}

On the basis of the International Society for the Study of Vascular Anomalies classification, vascular anomalies are categorized into vascular tumors and vascular malformations. Orbital vascular malformations are true developmental anomalies that can be further categorized according to the phenotypic types and flow dynamics. ${ }^{9,10}$ Distensible orbital venous malformations are sometimes referred to as orbital varices that demonstrate extensive connection to the venous vasculature. ${ }^{11}$ Surgical excision of venous malformation can be difficult because of its infiltrative nature, indistinct margins, and collapsible nature. Attempted direct excision can be complicated by severe intraoperative hemorrhage, which precludes complete excision. Embolization of the lesion prior to surgical excision has been demonstrated to facilitate resection with minimal morbidity. Injection of the embolizing agents can be performed under fluoroscopy guidance preoperatively before the patient is transported to the operating room or carried out intraoperatively with the conventional C-arm system. Couch et al reported successful surgical resection of orbital varices in four patients using preoperative fluoroscopy-guided embolization. Intraoperative fluoroscopy has been shown to be useful to delineate the full extent of the lesions. Lacey et $a l^{2}$ described the use of intraoperative percutaneous embolization guided by fluoroscopy during surgical excision of distensible orbital venous malformations in six patients. Using the same technique, Garcia et al ${ }^{12}$ were able to demonstrate complete surgical excision in three patients with anterior orbital venous malformations. Moreover, Tsai et al ${ }^{4}$ described a case of an orbital venous lesion that was exposed surgically and underwent intraoperative intralesional embolization before resection. In these studies, conventional fluoroscopy was employed, whereas in our current study a BDSAS capable of obtaining frontal and lateral projections simultaneously during a single injection of contrast was used. The advantage of BDSAS is that it allows simultaneous biplane imaging, thus permitting a high-quality three-dimensional assessment of the vascular lesion. Moreover, feeder vessels and outflow vessels are more clearly shown. Digital subtraction imaging is possible, and this reduces radiation exposure and interference by bone shadows. Better road mapping is also possible, which allows safer canalization and 

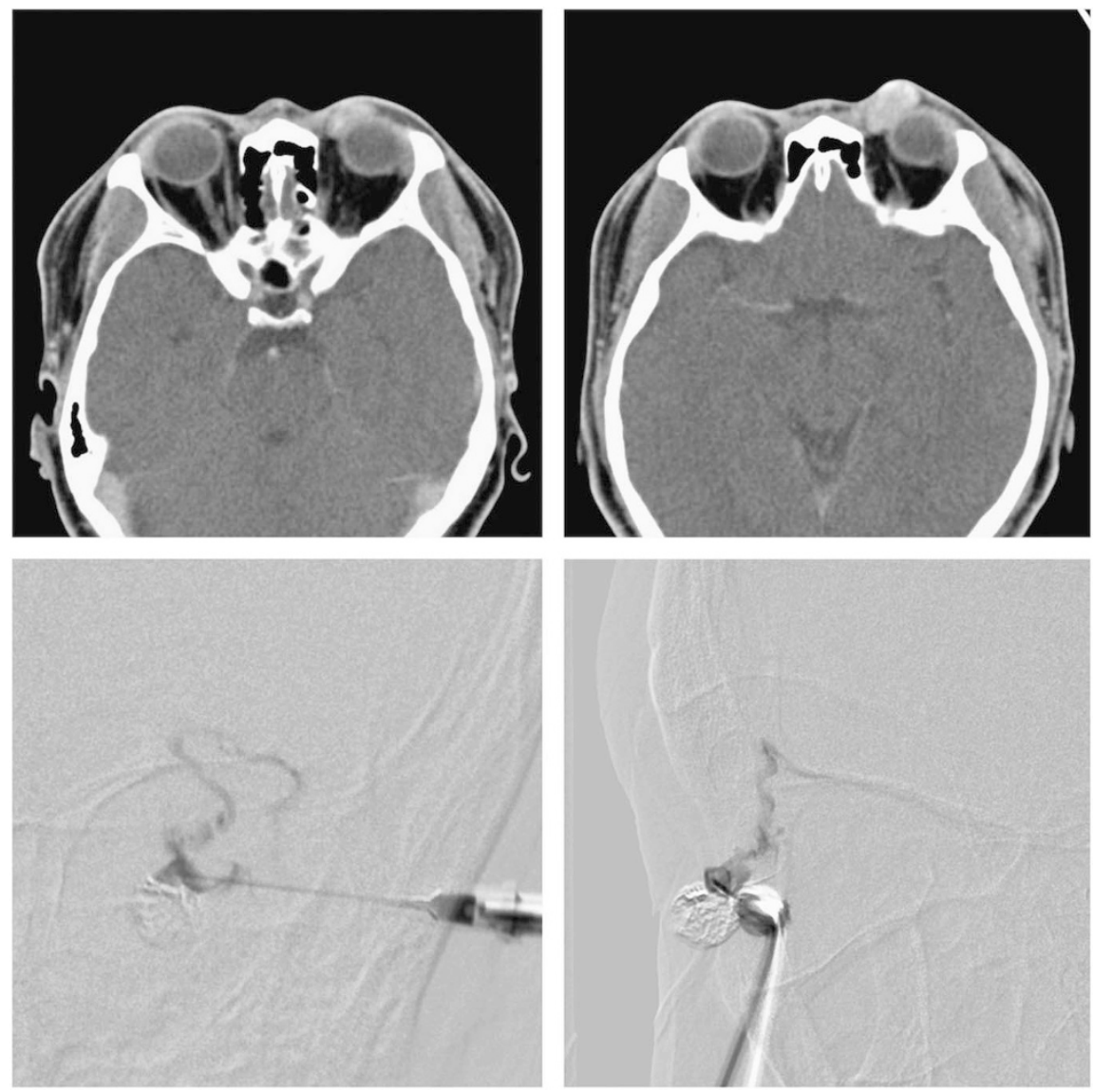

Figure 4 Clinical photos of Case 5. (Top left) Dynamic contrast-enhancing computed tomography showing the left upper lid venous malformation before valsalva maneuver. (Top right) Dynamic contrast-enhancing computed tomography showing the distensible nature of the same venous malformation after Valsalva maneuver. (Bottom left) Injection of tissue glue and contrast mixture was visualized and localized with the anterior-posterior view under the biplanar digital subtraction angiographic system. (Bottom right) The corresponding lateral view under the biplanar angiographic system.

embolization of the vascular lesion such that accidental intracranial glue injection via the ophthalmic veins can be avoided. Furthermore, digital recording and instant review is possible, which facilitates delineation of the more complicated or deep-seated lesions.

According to our experience, the hybrid procedure combining embolization and surgical resection under BDSAS guidance in the EVOR is safe and effective in managing orbital venous malformations. The setting of the EVOR in which facilities and equipment for interventional radiologists and orbital surgeons are available allows a one-stop provision of diagnosis and treatment of these complex vascular lesions. Real-time controlled injection of embolizing agents into the vascular lesion enhances the safety of the embolization procedure. As the orbit is an enclosed space, complete glue embolization of the venous malformation will cause elevated intraocular pressure or even result in compartment syndrome and vascular compromise, particularly for large distensible venous malformations. In the EVOR, the lesion is resected immediately after the glue injection, and the orbital pressure is relieved during orbitotomy; the duration of elevated orbital pressure is thus markedly reduced. In addition, the readiness of supplementary glue injection during surgery is important in multi-loculated or deep-seated lesions. For deep orbital vascular lesions, surgical dissection prior to glue injection allows open exposure of lesions for direct puncture, facilitating localization and embolization of the vascular abnormality. It is also possible to convert an unsuccessful percutaneous approach to an open surgical exposure in the setting of EVOR. Furthermore, suboptimal results or complications of the endovascular procedure like stenosis, occlusion, bleeding, and the development of compartment syndrome can be treated with immediate surgery, which improves the outcome of the procedure. With these dynamic supplementary procedures by interventional radiologists and orbital surgeons, more 
complex cases can be treated as the EVOR can handle both endovascular and open procedures in the same setting without transfer of the patient.

In conclusion, the hybrid procedure of orbital venous malformation in the EVOR is a novel application in ophthalmology. It is a safe and well-controlled procedure with real-time high-quality surveillance. Surgical resection is facilitated following direct intraoperative mapping and glue embolization. Overall, the surgical outcomes are good and the patient experience is satisfactory.

\section{Summary}

\section{What was known before}

- Surgical resection of orbital venous malformation guided by a conventional single-plane imaging system is difficult.

What this study adds

- The hybrid procedure of orbital venous malformation in the endovascular operating room is a novel application in ophthalmology.

- It is a safe and well-controlled procedure with real-time high-quality surveillance.

\section{Conflict of interest}

The authors declare no conflict of interest.

\section{References}

1 Harris GJ. Orbital vascular malformations: a consensus statement on terminology and its clinical implications. Orbital Society. Am J Ophthalmol 1999; 127: 453-455.
2 Lacey B, Rootman J, Marotta TR. Distensible venous malformations of the orbit: clinical and hemodynamic features and a new technique of management. Ophthalmology 1999; 106: 1197-1209.

3 Arat YO, Mawad ME, Boniuk M. Orbital venous malformations: current multidisciplinary treatment approach. Arch Ophthalmol 2004; 122: 1151-1158.

4 Tsai AS, Fong KS, Lim W, Al Jajeh I, Chuah CT, Rootman J. Bilateral orbital varices: an approach to management. Ophthal Plast Reconstr Surg 2008; 24: 486-488.

5 Couch SM, Garrity JA, Cameron JD, Cloft HJ. Embolization of orbital varices with $\mathrm{N}$-butyl cyanoacrylate as an aid in surgical excision: results of 4 cases with histopathologic examination. Am J Ophthalmol 2009; 148: 614-618.

6 Calligaro KD, Dougherty MJ, Patterson DE, Raviola CA, DeLaurentis DA. Value of an endovascular suite in the operating room. Ann Vasc Surg 1998; 12: 296-298.

7 Kpodonu J. Hybrid cardiovascular suite: the operating room of the future. J Card Surg 2010; 25: 704-709.

8 Murayama Y, Arakawa H, Ishibashi T, Kawamura D, Ebara M, Irie $\mathrm{K}$ et al. Combined surgical and endovascular treatment of complex cerebrovascular diseases in the hybrid operating room. J Neurointerv Surg 2013; 5: 489-493.

9 Rootman J. Vascular malformations of the orbit: hemodynamic concepts. Orbit 2003; 22: 103-120.

10 Rootman J, Heran MK, Graeb DA. Vascular malformations of the orbit: classification and the role of imaging in diagnosis and treatment strategies. Ophthal Plast Reconstr Surg 2014; 30: 91-104.

11 Heran MK, Rootman J, Sangha BS, Yeo JM. Dynamic arterialand valsalva-augmented venous phase multidetector CT for orbital vascular lesions: a pictorial review. Ophthal Plast Reconstr Surg 2014; 30: 180-185.

12 Garcia DD, Heran MK, Amadi AJ, Rootman J. Low outflow distensible venous malformations of the anterior orbit: presentation, hemodynamic factors, and management. Ophthal Plast Reconstr Surg 2011; 27: 38-43. 